\title{
Situaciones cotidianas anómalas y afectos negativos en el Perú
}

\author{
Ramón León ${ }^{1}$ \\ Universidad de Lima
}

\begin{abstract}
Se investigó a 479 universitarios peruanos (188 hombres y 291 mujeres) a través de una autoevaluación sobre la frecuencia de afectos y conductas negativas en ellos mismos, en su vecindad y en la sociedad peruana, y la de situaciones anómalas en su vida cotidiana. Agresiones verbales al otro y hablar mal de los demás obtuvieron los promedios más elevados en la valoración de los afectos y las conductas negativos en el barrio, pero la solidaridad fue asimismo vista como muy elevada. En la sociedad peruana, la envidia y la agresión verbal fueron evaluadas como las más altas y los promedios de los afectos positivos (solidaridad y confianza en los demás) fueron los más bajos. Ser víctima de un robo, ser víctima de un engaño y ser insultado obtuvieron los promedios más altos en situaciones anómalas. El resentimiento y la cólera fueron los afectos negativos de mayor magnitud.
\end{abstract}

Palabras clave: resentimiento, cólera, solidaridad, universitarios, conductas negativas, agresión.

\section{Abnormal daily situations and negative affects in Peru}

Four hundred seventy nine Peruvian students (188 male and 291 female) rated the frequency of negative social emotions in themselves, in their neighborhood, and in the Peruvian society; and the frequency of anomalous daily situations. Verbal aggressions and gossiping obtained the highest averages in the evaluations of affects in the neighborhood, but solidarity was rated as high than the negative affects. In the Peruvian society, envy and physical aggression were rated as the highest, and the positive affects obtained the smallest averages. To be a victim of an assault, to be cheated, and to be insulted obtained the highest averages in anomalous situations. Resentment and anger were the most salient negative affects.

Key words: resentment, anger, solidarity, students, negative conducts, aggression.

1 Licenciado en Psicología (Universidad Garcilaso de la Vega) y Dr. en Filosofía en la esp. de Psicología (Würzburg Universität, Alemania Federal, 1983). Profesor en las universidades Ricardo Palma y la Universidad de Lima. Autor de varios libros y Secretario de Redacción de la Revista de Neuro-psiquiatría. Rleon@correo.ulima.edu.pe. 

Mientras que la imagen exterior del Perú cambió favorablemente desde los desesperados años ochenta (en especial, los últimos, en que era visto como una nación al borde del caos y la hecatombe; e.g. Flores Galindo, 1988; Matos Mar, 1987; Stein y Monge, 1988; Tello, 1989) hasta hace poco, en que se solía enfatizar la estabilidad y tranquilidad alcanzadas (Youngers, 1999-2000), la percepción que los peruanos tienen de su país y, en particular, de su vida social ha cambiado relativamente poco, y continúa siendo escéptica, profundamente negativa.

En fecha reciente, una estudiosa anotaba en la mayoría de los peruanos la sensación de impotencia para influir en la toma de decisiones que resuelvan de verdad los problemas que los afectan (Lora, 2000). «El no-empleo, la incertidumbre del futuro y la pobreza que amenaza este futuro, la poca calidad de servicios que en otro tiempo fueron garantía de mejora social y económica como la educación, son temas muy presentes en la conciencia de nuestra población», señalaba (p. 35), aludiendo al efecto desmoralizador de esto en las personas.

La sensación de vivir en una sociedad democrática, fundamental en las sociedades modernas, es muy precaria en el Perú. Por el contrario: una atmósfera de desigualdad y de rotundas e insalvables diferencias continúa siendo característica de la sociedad peruana. El historiador Alberto Flores Galindo (1988) escribía que «todos saben que tanto en las decisiones importantes como en la vida cotidiana no todas las opiniones tienen el mismo valor: depende de quién las diga, cuál sea su aspecto físico, cómo se apellide, quiénes sean sus parientes $y$, desde luego, en qué nivel económico se ubique» (p. 24). 
Se escucha con frecuencia, en conversaciones informales o como observaciones incidentales en reuniones académicas, que en el Perú hay mucha envidia e hipocresía, que «aquí no se puede confiar en nadie», que la justicia es injusta, etc. «La frustración, el desánimo, la ausencia de esperanza son sentimientos frecuentes en la escritura peruana», insiste el ya citado Flores Galindo (1997, p. 327). Pero no sólo en ella, podríamos agregar; también en la vida cotidiana, como lo ponen en evidencia los escritos contenidos en un libro que, sintomáticamente, lleva por título En qué momento se jodió el Perú (Milla Batres, 1990).

Nuevas quejas se han agregado a las anteriores (que son sempiternas), como por ejemplo el reconocimiento de que en la sociedad peruana existe un marcado racismo, por lo general soterrado, pero que se expresa en ocasiones de modo claro y casi brutal (Manrique, 1996). Y no sólo el racismo, también el autoritarismo (Pásara y Parodi, 1988) y la violencia estructural (Portocarrero, 1990; Asociación Peruana de Estudios e Investigaciones para la Paz, 1993).

El propósito del trabajo fue explorar la valoración que tienen los jóvenes limeños sobre la realidad social peruana, tratando de conocer la ocurrencia en la vida de ellos de situaciones sociales anómalas y los afectos éstas han provocado. Asimismo, se trató de indagar qué importancia concedían ellos a los afectos negativos en la situación de subdesarrollo del país.

El estudio ha intentado vincular el interés por la realidad cotidiana con la exploración de la psicología de las emociones, dos áreas hasta ahora omitidas por negligencia en la investigación psicológica y psicosocial en general.

El estudio de lo cotidiano fue considerado como poco importante por psicólogos e investigadores de la realidad social. Se ha señalado en otra parte (León, Reyes y Vela, 2000) que lo cotidiano no encuentra un lugar en los libros dedicados a la historia del Perú; 
es como si no importara o no existiera. Pero lo cierto es que lo que sucede todos los días (o lo que no sucede) estructura, da sentido y contenido a la vida de las personas (Lora, 1994).

Lo cotidiano, entre tanto, se ha vuelto un concepto clave de la sociología (Elias, 1998). Estudiarlo es examinar la trama vital diaria, aparentemente sin mucho valor y más bien anecdótica, pero que es, sin embargo, la base sobre la cual se construye y elabora la vida de las personas y la dinámica social que termina siendo objeto de interés para sociólogos o antropólogos (Lora, 1994). En el Perú estos estudios recién comienzan (e. g. Panfichi y Portocarrero, 1995; Santos Anaya, 1999).

De más está decir que la vida cotidiana es también de interés para los psicólogos: «la realidad de la vida cotidiana es algo que cada uno comparte con los otros en un intercambio continuo entre su experiencia y la de los demás, donde cada uno cree, supone, infiere, confía o sospecha, donde uno puede desde encontrarse bien hasta vivir atormentado por sus fantasías de la experiencia, por los motivos y las intenciones de los otros, determinando con ello el modo de actuar» (Rovaletti, 2000; p. 212).

Las emociones, por su parte, han sido un área abandonada por los psicólogos. Un halo de misterio, de impenetrabilidad e irracionalidad las rodeaba. Sólo en las últimas décadas se ha iniciado su estudio sistemático (Izard, 1991; Plutchik, 1997).

La vinculación entre la vida cotidiana y las emociones es evidente. La vida diaria tiene una relación dialéctica con la cultura, en la que influye pero a la que también refleja. Así, por ejemplo, el racismo en Alemania no sólo fue la ideología del régimen de Hitler (Bullock, 1991). Precisamente por serlo, esa ideología influyó de modo decisivo en la vida del ciudadano común y corriente, ejerciendo una clara política de discriminación hacia algunos, obligando a 
otros a la emigración y, por último, liquidando a más de seis millones de personas. Un espíritu particularmente sensitivo como el del romanista Viktor Klemperer ha dejado conmovedor testimonio de los cambios ocurridos en la vida diaria en Alemania tras la Machtergreifung (Klemperer, 1991; otros testimonios interesantes están en Kruse y Schmitt, 2000).

La sociedad puede ser definida como un grupo organizado de personas que conviven en un espacio físico determinado, que aceptan determinadas reglas mínimas, reconociéndose recíprocamente deberes y derechos como miembros de ese grupo. Los integrantes de una sociedad interactúan entre sí; es decir, desarrollan comportamientos hacia otras personas y, al hacerlo, provocan reacciones y afectos tanto en ellos como en aquellos con quienes interactúan.

¿Cómo interactúan los peruanos entre sí? Difícilmente se podría decir que interactúan en condiciones de igualdad, aun cuando el Perú se defina como una sociedad democrática, en la que todos tienen igual valor, iguales derechos e iguales deberes.

Esa igualdad es sólo teórica, pues la condición de ciudadanía no es compartida por todos. López Jiménez (1997) resumió esta situación al referirse a ciudadanos reales e imaginarios, e indicar que en el Perú los hombres disfrutan más de la condición de ciudadanía que las mujeres, los costeños más que los serranos, los hispanohablantes más que aquellos que hablan quechua. En resumen, la sociedad peruana es profundamente inequitativa y, por lo tanto, la interacción de la mayoría de sus integrantes está marcada por esa inequidad.

Esa inequidad se expresa en la discriminación en el acceso y el uso de bienes y servicios, lo cual constituye, según Margalit (1997), una forma de humillación. Esa inequidad, por otra parte, tiene su correlato en la presencia de afectos que surgen y se expresan en el contexto de la vida social de las personas. Afectos tales como la soberbia, la indignación, la humillación y el resentimiento (Hansberg, 1996). 
Varios trabajos han reportado que existe una profunda desconfianza entre los peruanos (Westacott, sin fecha), que éstos atribuyen a los demás mucha envidia (León y Moscoso, 1991; León y Martell, 1994) y que, por lo tanto, se cuidan mucho de entrar en una relación de confianza con otras personas; que se piensa que en el Perú hay mucha hipocresía (León, Delhonte, del Castillo y León, 1998), y mucho desprecio (León y Tan, 1998). En dos estudios recientes se encontró que las personas consideraban que en la sociedad peruana hay mucho racismo y que la ley no tiene igual valor para todos (León, 1998a; León et al., 2000).

Obviamente, se trata de generalizaciones desmedidas. Pero, es importante, sin embargo, reflexionar acerca de las consecuencias de ellas. En otro contexto se ha señalado que «oir en tantas bocas y tantas veces que en el Perú la gente es muy hipócrita, muy envidiosa y 'rajona' y que 'aquí no se puede confiar en nadie', es, en realidad, un escalofriante diagnóstico de carácter negativo acerca de la calidad de la vida de relación en nuestro país». «Lo mejor, lo más aconsejable que uno puede hacer es cuidarse de los demás; atribuir 'de entrada' a los otros malas (o, en el menos malo de los casos, segundas) intenciones. Si 'la gente en el Perú es muy hipócrita’ lo más aconsejable es que no seamos 'ingenuos' ni 'nos dejemos engañar' por los demás. Si 'en el Perú la gente es muy envidiosa' lo más apropiado es no despertar la envidia, no darles confianza ni permitirles acceso a nuestra intimidad, dado que este afecto está vinculado al desarrollo de conductas agresivas hacia el envidiado. Si 'en el Perú la gente es muy rajona', hay que tener miedo de ser el centro de la atención y evitar la expresión de puntos de vista individuales. Y, por último, si en el Perú 'no se puede confiar en nadie', entonces la desconfianza y el recelo se convierten en reglas de otro» (León y Tan, 1998).

Los afectos sociales negativos (como también los positivos) juegan un rol particularmente importante en la calidad de la vida social. Así, una persona envidiosa no podrá desarrollar una vinculación afectiva genuina con aquella a la que envidia (Castilla del Pino, 
1994); las relaciones entre aquellos que desprecian y los despreciados en modo alguno podrán ser de carácter positivo.

Entre los afectos sociales negativos más estudiados se encuentran la envidia y el resentimiento. La primera ha sido objeto de consideraciones filosóficas y morales (por ejemplo, Russell, 1978) e históricas (e.g. Milobenski, 1964; Walcot, 1978), aparte de reflexiones psicológicas como las de Melanie Klein (1976). En la última década del siglo XX los estudios e investigaciones se multiplicaron (Alberoni, 1991; Boris, 1993; Castilla del Pino, 1994; Girard, 1995; Salovey, 1991; Tomei, 1995), quizás porque la cultura contemporánea insiste en la igualdad de valía de las personas, pero siguen existiendo diferencias en ingresos, en posibilidades de desarrollo personal, en acceso a bienes y servicios, razón por la cual la envidia habría aumentado (Ciabattari, 1989).

El resentimiento es otra emoción social que ha sido objeto de numerosas consideraciones filosóficas (Nietzsche, 1983; Scheler, 1944), que lo definen como el sentimiento de que uno ha sido objeto de alguna injusticia, víctima de algún maltrato, o que la vida en general no lo ha tratado de modo equitativo (León y Romero, 1990). De acuerdo con Scheler (1944), comprende rencor y ánimo vindicativo, o sea deseos de revancha, impidiendo al individuo establecer vínculos positivos en el plano social y afectivo con los demás.

En su clásico libro La estructura de la personalidad, Lersch (1968) escribe: «El resentimiento se origina (...) siempre a partir del conocimiento de haber sido tratado peor que los demás, de haber salido perdiendo y de haber sido perjudicado por el destino, cuando este conocimiento se acompaña de envidia» (p. 143).

Obviamente, no se pueden dar cifras precisas, pero -como ya se anotó- es frecuente escuchar que en el Perú hay mucha envidia, mucha hipocresía, mucho resentimiento, que no se puede confiar en nadie y que la corrupción está generalizada Aunque estas son afirmaciones 
que deben ser tomadas cum grano salis, lo cierto es que la realidad cotidiana (con sus inequidades y carencias) puede justificar que ellas circulen y tengan tanta aceptación. Dos libros (Evaluación social del desarrollo humano en el Perú de Eguren, Cano y del Águila, 1997; y, Ciudadanos reales e imaginarios. Concepciones, desarrollo y mapas de la ciudadanía en el Perú de López Jiménez, 1997) hacen un dramático recuento de las amplias diferencias en ingresos, en educación, en acceso a servicios y en calidad de vida en general que se registran hoy en el Perú.

Como ya se dijo, sociedades profundamente inequitativas, en las cuales la opulencia y la miseria conviven -es decir, sociedades como la peruana-, son caldos de cultivo para afectos y conductas negativas (Alarcón, 1986; Buss, 2000).

La frustración, la indignación, el resentimiento, la envidia y el afán vindicativo cobran fuerza en contextos sociales caracterizados por la injusticia, en los que las personas se dan cuenta temprano de que a pesar de la predicada igualdad por parte del estado los individuos no son iguales, $\mathrm{o}$, como diría Orwell, unos son más iguales que otros.

Pero la vida cotidiana de la gran mayoría de peruanos no sólo está signada por la inequidad y la pobreza. También se registra en ella una gran cantidad de situaciones anómalas de variada naturaleza y expresión: desde insultos y ofensas personales hasta robos y asaltos, que son causa de incertidumbre en las personas y de que ellas redoblen las medidas de seguridad.

Engaños, estafas y abusos de confianza suelen ser denunciados o reportados por un elevado porcentaje de ciudadanos. También se escuchan quejas acerca de maltratos, discriminaciones, chismes, etc.

La influencia de estos sentimientos negativos y de estas conductas no menos negativas en la calidad de la vida social de los peruanos apenas ha sido explorada. Esto constituye una de las grandes 
deudas de las ciencias sociales. Recientemente, sin embargo, esta deuda ha comenzado a ser saldada, como lo demuestran algunos trabajos (APRODEH-CEDAL, 1996; Eguren et al., 1997; López Jiménez, 1997; Portocarrero, 1998; Twanama, 1992). Esta investigación se inscribe en esta corriente de estudio de los afectos de la cotidianeidad injusta del Perú.

\section{Metodología}

\section{Participantes}

La muestra estuvo conformada por 479 universitarios. Participaron 188 hombres $(39.2 \%)$ y 291 mujeres $(60.8 \%)$. Los estudiantes provenían de una universidad privada y de una universidad pública.

Los participantes residían en Lima. Al 3.8\% se le consideró de clase alta; $80.8 \%$ de clase media, y $13.4 \%$ de clase baja; $2.1 \%$ no se ubicó en ningún nivel. En cuanto a la asignación racial, al $27.1 \%$ de los sujetos se le consideró blanco; $65.6 \%$ mestizo; $2.5 \%$ andino; $1.5 \%$ negro; $y$, $2.1 \%$ oriental.

\section{Instrumentos}

-La Lista de Evaluación de Frecuencia de Afectos y Conductas (León, 2002).- Estaba conformada por ocho afectos y conductas negativos, y dos positivos. Los negativos fueron:

- $\quad$ sentir envidia

- experimentar resentimiento

- agredir verbalmente a otro (o ganas de hacerlo)

- agredir físicamente a otro (o ganas de hacerlo)

- mostrarse indiferente

- $\quad$ ser despreciativo

- tener prejuicios raciales

- hablar mal de los demás 
Los positivos fueron:

- confiar en los demás

- ser solidario

La persona tenía que responder con qué frecuencia ocurren esos afectos y conductas sociales en sí misma, en su barrio y en la sociedad peruana. Habían seis opciones de respuesta $(0=$ nunca, $1=$ casi nunca, $2=$ pocas veces, $3=$ con regular frecuencia, $4=$ con mucha frecuencia, 5 = siempre).

-La Lista de Situaciones Negativas (León, 2002).- Estaba conformada por diez ítems:

- pagar una coima

- $\quad$ ser discriminado por mi raza

- $\quad$ ser víctima de un robo

- $\quad$ ser objeto de chismes

- $\quad$ ser insultado

- $\quad$ sentir que se me desprecia

- no se reconocen mis méritos

- $\quad$ ser víctima de un engaño

- $\quad$ ser tratado con indiferencia

- ser agredido físicamente.

El evaluado tenía que responder en primer lugar si ha experimentado esas situaciones. Luego, en qué grado (desde $0=$ nada hasta $5=$ mucho) esas situaciones le han suscitado las siguientes reacciones: resentimiento, temor, cólera o vergüenza (ver anexo).

-Lista de sentimientos que contribuyen al subdesarrollo del Perú.- Esta lista incluía el resentimiento, la desconfianza entre los peruanos, la envidia, el racismo y la falta de solidaridad.

-Lista de causas del resentimiento y de la envidia en el Perú.Este cuestionario tomó en cuenta cinco posibilidades: diferencias 
económicas entre los peruanos; falta de oportunidades para los peruanos; los peruanos somos así; mucho racismo y discriminación entre los peruanos; y mucho desprecio entre los peruanos.

\section{Procedimiento}

Los universitarios respondieron de manera anónima y colectiva la Lista de Evaluación de Frecuencia de Afectos y Conductas, y la Lista de Situaciones Negativas.

\section{Resultados}

\section{Afectos y conductas}

El Cuadro 1 presentan los promedios en la evaluación de la frecuencia de los diez afectos y conductas llevada a cabo por los sujetos con respecto a ellos mismos, su barrio y la sociedad peruana. La Figura 1 ilustra los resultados.

\section{Cuadro 1}

Frecuencia de ocurrencia de los diez afectos y conductas con respecto a sí mismo, al barrio y a la sociedad peruana

\begin{tabular}{|lccc|}
\hline Afectos y conductas & ti mismo & Barrio & Soc. Peruana \\
\hline Envidia & 1.51 & 2.27 & 3.45 \\
Resentimiento & 1.80 & 2.13 & 3.20 \\
Agresión Verbal & 1.85 & 2.42 & 3.36 \\
Agresión Física & 1.06 & 1.83 & 3.02 \\
Indiferencia & 1.65 & 2.31 & 2.99 \\
Desprecio & .98 & 1.83 & 2.84 \\
Prejuicios sociales & .80 & 1.79 & 3.13 \\
Hablar mal de los demás & 1.19 & 2.40 & 3.32 \\
Confianza en los demás & 2.64 & 2.12 & 1.91 \\
Solidaridad & 3.21 & 2.54 & 2.36 \\
\hline
\end{tabular}


Situaciones cotidianas anómalas y afectos negativos en el Perú

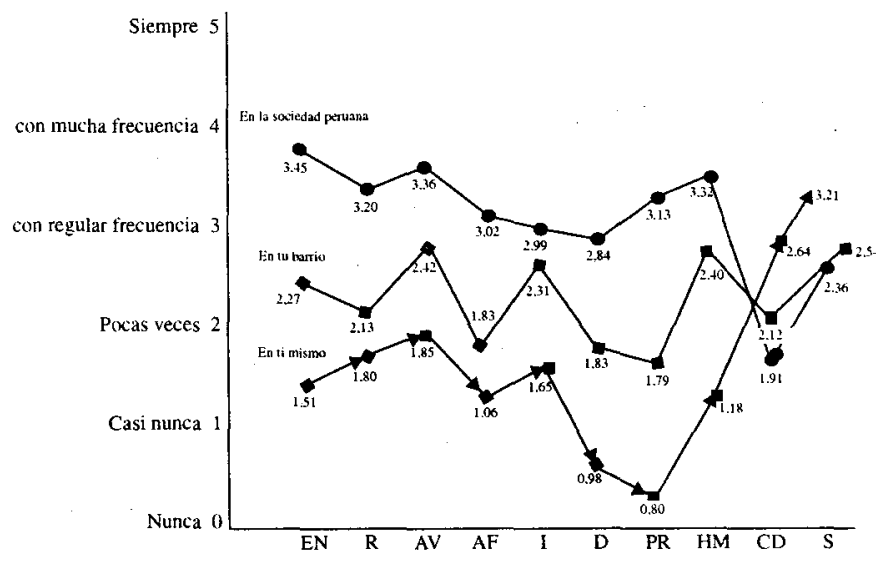

Figura 1. Promedios de la frecuencia de los diez afectos y conductas sociales en ti mismo, en tu barrio y en la sociedad peruana.

EN: envidia, RE: resentimiento, AV: agresión verbal, AF: agresión física, IN: indiferencia, DE: desprecio, PR: prejuicios raciales, HM: hablar mal de los demás, CO: confiar en los demás, SO: solidaridad.

Los afectos y conductas negativos recibieron bajas puntuaciones en el área en ti. Los más altos fueron los de resentimiento y agresión verbal ( 1.80 y 1.85 , respectivamente), mientras que los más bajos correspondieron a ser despreciativo (.98) y tener prejuicios raciales (.80). Los dos afectos positivos, tener confianza en los demás y ser solidario, obtuvieron por el contrario promedios elevados: $2.64 \mathrm{y}$ 3.21 , respectivamente.

En el área en tu barrio, agredir verbalmente al otro (2.42) y hablar mal de los demás (2.40) obtuvieron los promedios más elevados, seguidos por la indiferencia (2.31) y la envidia (2.27), entre los afectos negativos. Sin embargo, la valoración de solidaridad obtuvo el promedio más elevado (2.54).

Por último, en el área en la sociedad peruana, la envidia (3.45), seguida de la agresión verbal (3.36), obtuvieron los promedios más elevados. Hablar mal de los demás (3.32) los siguió. Los promedios 
de los afectos positivos fueron los más bajos (confianza en los demás: 1.91; solidaridad: 2.36 ) .

Como puede verse, en el caso de los afectos negativos la dirección ascendente de los promedios va del área en ti a en la sociedad peruana, mientras que en el de los positivos la dirección es a la inversa: los promedios más altos se presentaron en el área en tí y los más bajos ocurrieron en la sociedad peruana.

En cuanto a la segunda evaluación realizada, la frecuencia reportada de situaciones negativas es elevada (Cuadro 2). Algunas, que corresponden a hechos objetivos como ser víctima de un robo, han sido experimentadas por alrededor del $70 \%$ de los encuestados, según sus propios reportes. Algo semejante sucede en un porcentaje todavía mayor en lo referido a la situación ser víctima de un engaño (circa $75 \%$ ). Otra situación reportada como frecuente fue ser insultado, con un mayor porcentaje mayor de hombres $(80.31 \%)$ que de mujeres $(70.79 \% ; p<.019)$.

\section{Cuadro 2}

Frecuencias (\%) de Situaciones Anómalas en la sociedad peruana

\begin{tabular}{|c|c|c|c|c|c|c|}
\hline \multirow[t]{2}{*}{ Situación } & \multicolumn{2}{|c|}{ Hombres } & \multicolumn{2}{|c|}{ Mujeres } & \multirow[t]{2}{*}{$X^{2}$} & \multirow[t]{2}{*}{$p$} \\
\hline & Sí & No & Sí & No & & \\
\hline 1.Pagar una coima & 55.86 & 44.14 & 21.64 & 78.35 & 58.66904 & .000 \\
\hline 2. Ser discriminado & 21.80 & 78.20 & 17.90 & 82.10 & 1.13268 & .287 \\
\hline 3. Ser víctima de robo & 73.94 & 26.06 & 68.73 & 31.27 & 1.49741 & .221 \\
\hline 4. Ser objeto de chismes & 70.22 & 29.78 & 80.42 & 19.58 & 6.59164 & .010 \\
\hline 5. Ser insultado & 80.31 & 19.69 & 70.79 & 29.21 & 5.46301 & .019 \\
\hline 6. Ser despreciado & 42.55 & 57.45 & 44.32 & 55.68 & .14659 & .701 \\
\hline 7. No se reconocen mis méritos & 55.31 & 44.69 & 53.95 & 46.05 & .08610 & .769 \\
\hline 8. Ser víctima de un engaño & 76.06 & 23.94 & 74.91 & 25.09 & .08132 & .775 \\
\hline 9. Ser tratado con indiferencia & 55.31 & 44.69 & 62.54 & 37.46 & 2.47739 & .115 \\
\hline 10: Ser agredido físicamente & 56.38 & 43.62 & 25.08 & 74.92 & 47.79906 & .000 \\
\hline
\end{tabular}

Por otro lado, la situación reportada como menos frecuente fue ser discriminado: alrededor del $20 \%$ aceptó haberla experimentado. 
Situaciones cotidianas anómalas y afectos negativos en el Perú

Pagar coima fue reportada por más del doble de hombres que mujeres $(55.86 \%$ vs. $21.64 \% ; p<.00)$. Algo semejante ocurrió con ser agredido físicamente $(56.38 \%$ de varones vs. $25.08 \%$ de mujeres; $p<.00)$.

Situaciones que suponen una valoración más subjetiva fueron las de ser despreciado y ser tratado con indiferencia. Con respecto a la primera, $42.55 \%$ de varones y $44.32 \%$ de mujeres afirmaron que les había ocurrido. Acerca de la segunda, $55.31 \%$ de varones y $62.54 \%$ de mujeres la reportaron.

Aún más subjetiva es la situación ser objeto de chismes, reportada por $70.22 \%$ de varones y $80.42 \%$ de mujeres $(p<.01025)$. Ser insultado, por su parte, fue una situación más informada por varones que por mujeres $(80.31 \%$ vs. $70.79 \%$ de mujeres, $p<.019$ ).

Ser despreciado, no se reconocen mis méritos y ser tratado con indiferencia no arrojan diferencias significativas por sexo, pero tuvieron una frecuencia más bien elevada: cuatro de cada diez sujetos de la muestra señalaron haber sido despreciados, cinco afirmaron que no se les reconocieron sus méritos y cinco hombres y seis mujeres se quejaron de haber sido tratados con indiferencia

En cuanto a los afectos causados por situaciones sociales negativas, el Cuadro 3 resume los resultados de la evaluación de los afectos provocados por las situaciones sociales negativas. 


\section{Cuadro 3}

Promedios de los afectos provocados por las situaciones sociales negativas según sexo

\begin{tabular}{|lclllllll|}
\hline \multicolumn{1}{|c}{ Situación } & \multicolumn{2}{c}{ Resentimiento } & \multicolumn{2}{c}{ Temor } & \multicolumn{2}{c|}{ Cólera } & \multicolumn{2}{c|}{ Vergüenza } \\
& $\mathrm{H}$ & $\mathrm{M}$ & $\mathrm{H}$ & $\mathrm{M}$ & $\mathrm{H}$ & $\mathrm{M}$ & $\mathrm{H}$ & $\mathrm{M}$ \\
\hline 1. Pagar una coima & 1.78 & 1.80 & 1.85 & $2.36^{* * *}$ & 2.54 & $2.85^{*}$ & 2.21 & $2.68^{* *}$ \\
2. Ser discriminado & 2.02 & $2.57^{* * *}$ & 1.44 & 1.44 & 2.50 & $3.15^{* * *}$ & 1.47 & 1.69 \\
3. Ser víctima de robo & 2.62 & 2.45 & 2.31 & $3.25^{* * * *}$ & 3.82 & 3.78 & 1.56 & $1.29^{*}$ \\
4. Ser objeto de chismes & 2.36 & $2.94^{* * *}$ & 1.41 & 1.62 & 2.91 & $3.65^{* * * *}$ & 1.35 & 1.53 \\
5. Ser insultado & 2.42 & $2.95^{* * *}$ & 1.48 & 1.57 & 3.27 & $3.59^{*}$ & 1.56 & $1.95^{* *}$ \\
6. Ser despreciado & 2.35 & $2.68^{*}$ & 1.62 & 1.69 & 2.32 & $2.77^{* *}$ & 1.55 & 1.69 \\
7. No se reconocen mis méritos & 2.37 & $2.83^{* * *}$ & 1.50 & 1.45 & 2.60 & $3.03^{* * *}$ & 1.31 & 1.34 \\
8. Ser víctima de un engaño & 2.88 & $3.35^{* * *}$ & 1.66 & $1.98^{* *}$ & 3.33 & $3.79^{* *}$ & 1.75 & 1.87 \\
9. Ser tratado con indiferencia & 2.42 & $2.85^{* * *}$ & 1.62 & 1.73 & 2.60 & $2.93^{*}$ & 1.68 & 1.71 \\
10. Ser agredido físicamente & 2.44 & $2.75^{*}$ & 1.95 & $2.48^{* * *}$ & 3.37 & 3.35 & 1.79 & 1.82 \\
\hline
\end{tabular}

Nota. H: Hombres, M: Mujeres. $\quad * p<.05 \quad{ }^{* *} p<.01 \quad * * * p<.005 \quad * * * * p<.000$

El resentimiento alcanzó los valores más elevados en la situación ser víctima de un engaño (H: $2.88 ; \mathrm{M}: 3.35 ; p<.005)$, situación que asimismo aparece asociada a promedios mayores de cólera $(\mathrm{H}$ : 3.33 ; $\mathrm{M}: 3.79 ; p<.01)$, junto con ser víctima de un robo $(\mathrm{H}: 3.82$; M: 3.78). El temor tuvo sus promedios más elevados (tanto en hombres como en mujeres) precisamente cuando se piensa en la posibilidad de ser víctima de un robo (H: $2.31 ; \mathrm{M}: 3.25 ; p<.00)$. Fue otra, sin embargo, la situación que causó vergüenza: la de pagar una coima (H: 2.21; M: 2.68; $p<.01$ ).

Obsérvese, por último, que en la mayoría de las situaciones los valores de cada uno de los afectos fueron más elevados en las mujeres que en los varones. Ser objeto de chismes y ser discriminado por mi raza son un buen ejemplo de esto: tanto en resentimiento como en cólera los promedios de ellas son más altos. Hay dos excepciones, sin embargo: ser víctima de un robo causó más resentimiento, cólera y vergüenza en los hombres, así como ser agredido físicamente estuvo asociado con más cólera. 
Situaciones cotidianas anómalas y afectos negativos en el Perú

En la siguiente Figura se presenta la valoración de los sujetos de la importancia de los sentimientos negativos en la situación de subdesarrollo del Perú.

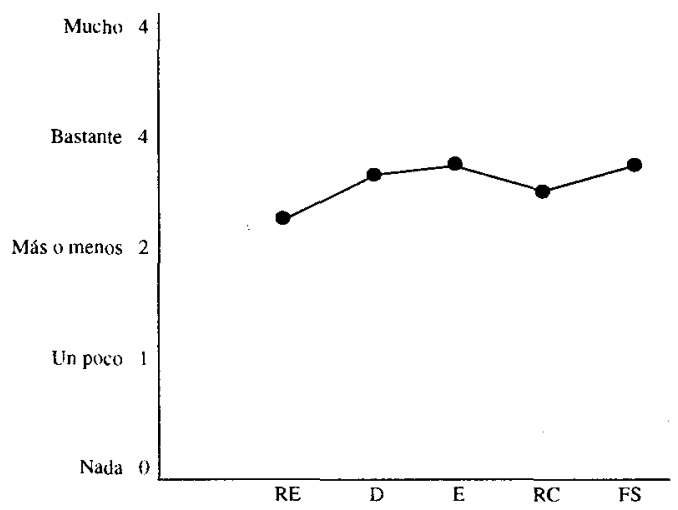

Figura 2. Valoración de la importancia de los sentimientos negativos en el subdesarrollo del Perú.

RE: resentimiento, D: desconfianza ante las personas, E: envidia, RC: racismo, FS: falta de solidaridad.

La falta de solidaridad y la envidia tuvieron los promedios más elevados ( 2.73 en ambos casos), seguidos por la desconfianza entre los peruanos (2.70) y luego por el racismo (2.63).

Con respecto al resentimiento, el racismo o y la discriminación fueron las causas más valoradas (5.44), seguidas por la falta de oportunidades (5.39) y el desprecio (5.26). En lo que se refiere a la envidia, la causa más valorada fue diferencias económicas (5.70), seguida por el racismo y la discriminación (5.33) y por la falta de oportunidades (5.29). La Figura 3 resume los resultados acerca de las causas del resentimiento y de la envidia en el Perú. 


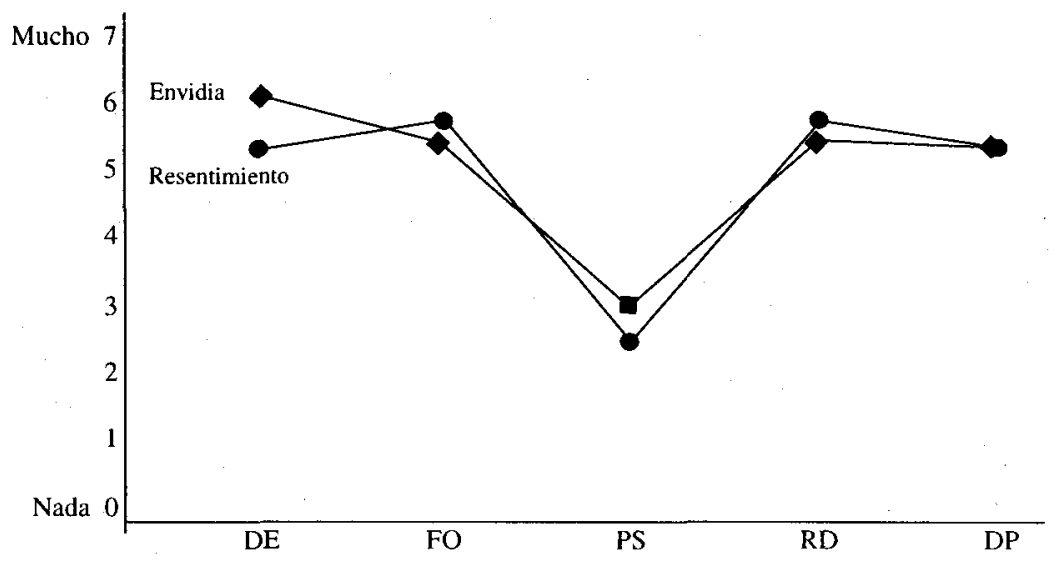

Figura 3. Causas del resentimiento y la envidia en el Perú.

DE: diferencias económicas entre los peruanos, FO: falta de oportunidades, PS: los peruanos somos así, RD: racismo y discriminación, DP: desprecio.

\section{Discusión}

Se observó en los resultados que, si bien los sujetos se consideraban a sí mismos como poco envidiosos, poco resentidos, poco chismosos y poco discriminadores, también consideraron que en su barrio y, muy en especial, en la sociedad peruana la envidia, el chisme y la discriminación son realidades de gran frecuencia.

Esto no debe sorprender, pues las características poco deseables en el plano social suelen por lo general ser rechazadas por los que tienen que evaluarse en ellas (Banaji y Prentice, 1994). Ser envidioso o ser discriminador son características poco deseables, mal vistas en la sociedad. La primera -ser envidioso- supone reconocer carencias o admitir inferioridades, algo que atenta contra la autoimagen (Castilla del Pino, 1994; Foster, 1972). La segunda -ser discriminador o practicar la discriminación- se ha convertido en inadmisible en las sociedades modernas (Callirgos, 1993; Delacampagne, 1983; León, 1998a). 
Situaciones cotidianas anómalas y afectos negativos en el Perú

Estos resultados aceptan dos alternativas de interpretación. De un lado, se puede destacar las limitaciones de los reactivos de autorreporte, que están a merced de la posibilidad de que las personas falseen sus respuestas o las hagan según un sesgo que sea, a su vez, la expresión de un rasgo de personalidad. Así, por ejemplo, Furnham, Borovoy y Henley (1986) demostraron que personas que tienen un patrón de conducta Tipo A tendían a autovalorarse más negativamente que las del tipo B. Por lo general, se controla esta posibilidad por medio de escalas de validez o de mentiras. En la presente investigación no se empleó una escala de esta índole.

La otra posibilidad se refiere al hecho de que las personas que han respondido a los reactivos aquí utilizados tengan como una de sus características el ser poco críticas acerca de su propio comportamiento. Desde González Prada (1976) hasta nuestros días, los estudiosos de la sociedad peruana han destacado que la ausencia de autocrítica, la carencia de evaluación objetiva de las situaciones externas y de las propias circunstancias, así como la aceptación de situaciones anómalas casi como elemento normal de la realidad, son el denominador común de la sociedad peruana (Nugent, 1992; Portocarrero, 1990; Twanama, 1992). Así, en una reciente apreciación de la realidad social peruana se puede leer lo siguiente: «la población termina por aceptar como normal que se ejercite el acto de corrupción entre un policía y el chofer de un microbus, por ejemplo, o entre un litigante y algún funcionario del juzgado correspondiente, si de esa forma uno evita una papeleta y el otro se salva de un engorroso proceso u obtiene la copia de un documento más o menos importante» (Pedraglio, 2001, p. 60).

Son los mismos sujetos que se consideran a sí mismos como poco envidiosos, poco discriminadores y poco chismosos, los que por lo general señalan que la discriminación, la envidia y la maledicencia suelen ser frecuentes en sus barrios y en la sociedad peruana. La Figura 1 pone esto en evidencia. 
Por ello es posible afirmar que la imagen que tienen los sujetos es la de una sociedad que conserva algunos rasgos casi feudales como la discriminación (es decir, la aceptación de diferencias sustantivas en el valor de las personas, y la prácticas de conductas diferenciales hacia ellas) y en la cual la calidad de las relaciones sociales está emponzoñada por antivalores (esta palabra no es castellano), en antagonismo con los rasgos de una sociedad moderna (Callirgos, 1997).

Esta visión negativa de la sociedad peruana explica los elevados promedios de agresión verbal y de resentimiento que los propios sujetos admiten en ellos mismos.

La percepción que los sujetos tienen de su entorno inmediato (en tu barrio) no es menos negativa: aunque la solidaridad obtiene el promedio más elevado, la agresión verbal y hablar mal de los demás la siguen de cerca. La confianza en los demás tiene uno de los promedios más bajos.

En lo que se refiere a la sociedad peruana, la envidia, la agresión verbal, hablar mal de los demás y el resentimiento son las emociones percibidas como más frecuentes. La solidaridad y, sobre todo, la confianza en los demás, reciben los promedios más bajos.

Esto es: para los sujetos investigados en la sociedad peruana la envidia, la discriminación, la agresión verbal y hasta la agresión física suelen ser frecuentes; por ello, difícilmente podrá ser considerada una sociedad democrática y moderna, o, como lo dice Margalit (1997), una sociedad decente.

La información acerca de la calidad de las relaciones sociales se encuentra en el Cuadro 2. Con excepción del ítem 2 (ser discriminado), los porcentajes afirmativos van de moderados a elevados para uno y otro sexo, en especial entre los varones. Entre las mujeres, 
además del ítem 2, otros con porcentajes afirmativos relativamente bajos fueron pagar una coima y ser agredido físicamente.

Si nos guiamos por los porcentajes afirmativos, ser víctima de un robo, ser víctima de engaños y ser insultado son situaciones sociales negativas de alta frecuencia en la vida de ellos.

En lo referente a ser insultado, los resultados confirman los encontrados por Anicama, Vizcardo, Carrasco y Mayorga (1999), que nos informan además de algo que no fue averiguado en el presente estudio: ¿de dónde provienen los insultos? Ellos encontraron que uno de cada cuatro encuestados reportaba haber sido insultado por sus padres, y uno de cada cinco por sus amigos.

La frecuencia elevada de ser insultado debe ser entendida en su real sentido, que es dramático. Margalit (1997) anota que «las palabras insulto y humillación se hallan en un continuum», y que por insulto se entiende «una herida al honor social» (p. 104).

La afirmación de Margalit se vincula, a través de los resultados de este estudio, a la presencia de una experiencia que, según los historiadores, es muy frecuente en la sociedad peruana: la de la humillación (Manrique,1999). La humillación, en las palabras de Margalit, «lesiona el valor intrínseco de uno mismo» (p. 104).

Un hecho que resulta un indicador alarmante acerca del modo en que los jóvenes perciben la sociedad peruana es que uno de cada dos afirma haber sido despreciado (ítem 6), algo que confirma lo que el autor encontró en otro contexto (León y Tan, 1998), y que guarda relación con el elevado porcentaje de los que encuentran que la sociedad peruana no los estimula, pues no reconoce sus méritos (ítem 7).

Los psicólogos sociales y los del desarrollo (Harré, 1982; Horrocks; 1984) han destacado la importancia que los adolescentes y los 
jóvenes conceden a la adecuada valoración de sus méritos y de sus logros por parte de los demás, y el significado negativo que tiene en ellos la ausencia de dicha valoración. Los resultados deben ser considerados preocupantes. Como en otra parte se ha señalado (León, 1998 b), la ausencia de reconocimiento tiende al desarrollo de sentimientos sociales negativos hacia los demás.

Son, por cierto, tanto más preocupantes cuanto que provienen de universitarios. Es decir, de personas que esperan ser admitidas por la sociedad peruana en base a la educación, una de las vías legítimas de ascenso social (Ansión, Lazarte, Matos, Rodríguez y Vega-Centeno, 1998), pero que al salir de las aulas descubrirán que eso no siempre es posible por el autoritarismo y el racismo predominantes en el Perú (Flores Galindo, 1988). Son precisamente el racismo, la discriminación y la falta de oportunidades las que causan más resentimiento $y$ envidia.

En lo referido a los sentimientos causados por situaciones sociales anómalas, la cólera y el resentimiento son los afectos de mayor intensidad, tanto en uno y en otro sexo. Este binomio afectivo da un tono negativo a la vida de relación social de las personas y se erige como un severo obstáculo en el proceso de identificación de ellas con la sociedad peruana. Si se considera que más del $70 \%$ de los sujetos afirmó haber sido víctima de un engaño, así como los porcentajes elevados de quienes dicen haber sido insultados y objeto de chismes, es posible reconocer la frecuencia y la intensidad preocupantes de la cólera y del resentimiento, que traen como consecuencia irritabilidad, hostilidad, tendencia a la agresión (Plutchik, 1997).

De otro lado, en la explicación del subdesarrollo en el Perú los sujetos atribuyen un peso mayor a la envidia y a la desconfianza entre los peruanos, así como a la falta de solidaridad entre ellos.

El resentimiento, según lo reportado por los sujetos (Figura 3) tiene sus causas en el racismo y en la discriminación, así como en la 
falta de oportunidades, situaciones todas de elevada frecuencia en el Perú, un país en el cual la desocupación es un mal endémico y en el que el desprecio es una experiencia muy difundida (Nugent, 1992). Por su parte, las diferencias económicas se encuentran en el origen de la envidia.

«Nunca ha sido fácil vivir en el Perú», escribió alguna vez Flores Galindo (1988; p. 232). Su afirmación conserva plena vigencia hoy. Los resultados apuntan a una frecuencia e intensidad elevadas de emociones negativas entre los encuestados que los lleva a ver la vida en el Perú como llena de riesgos y dificultades, y en la cual predominan la falta de solidaridad, la envidia y la desconfianza interpersonal (Figura 1). Estos tres sentimientos, a su vez, son vistos como los principales problemas para alcanzar el desarrollo del país.

Comentando una encuesta que tuvo por título ¿Por qué no vivo en el Perú?, Gustavo Buntinx señala que una de las razones que llevaron a sus encuestados (intelectuales, artistas y profesionales) a alejarse del país fue la pobreza ética y política, mucho más gravitante aún que la material. «El racismo, la iniquidad y la arrogancia social contaminan, envenenan y pervierten las relaciones sociales entre nosotros», afirma (Buntinx, 1998). A ellos habría que agregar los robos, engaños, insultos, chismes y discriminaciones.

Si en los años ochenta la vida cotidiana de los peruanos fue marcada a fuego por el terrorismo, la inflación desbordada, la falta de agua y de luz y la corrupción, la década que acaba de terminar no tiene rasgos tan dramáticos (excepto en el caso de la corrupción), pero ofrece un perfil de inseguridad frente al robo, de marcada desconfianza en las relaciones interpersonales como resultado de la atribución a los demás de segundas intenciones y de maledicencia; de proclividad a la agresión verbal en la forma del insulto, e, inclusive, a la agresión física. 
La imagen que se configura a partir del examen de los resultados confirma el escepticismo y la desconfianza mencionados por la mayoría de los peruanos al momento de enjuiciar la realidad social de su país. Por cierto, otros trabajos apuntan en esa misma dirección (Anicama et al., 1999; Eguren et al. 1997; Portocarrero, 1998).

Una consideración aparte merecen los bajos promedios obtenidos en los dos sentimientos positivos de la Lista de Evaluación de Frecuencias y Conductas Sociales: la confianza en los demás y la solidaridad.

Uno y otro son afectos centrales en la vida social, pues definen la calidad de las relaciones sociales de las personas que forman parte de un grupo. Mientras que el sentimiento de solidaridad no ha sido explorado de modo específico en el Perú, estudios y reflexiones que tienen como objeto el racismo y a la exclusión social (León, 1998a; León y Tan, 1998; León et al., 2000; Portocarrero, 1993; Twanama, 1992; Carranza, 2000) permiten afirmar que la solidaridad entre los peruanos es muy precaria.

La confianza y, en particular, la confianza interpersonal sí ha sido evaluada en fecha reciente (Romero, 2001; Romero y Sulmont, 2000). En una de esas evaluaciones se encontró que sólo el $5 \%$ de los peruanos declaraba que se podía confiar en otros. Un resultado que se ve confirmado por los hallazgos reportados en esta comunicación.

\section{Referencias}

Alarcón, R. (1986). Psicología, pobreza y subdesarrollo. Lima: INIDE.

Alberoni, F. (1991). Gli invidiosi. Milán: Garzanti.

Anicama, J., Vizcardo, S., Carrasco, J. y Mayorga, E. (1999). Estudio epidemiológico sobre la violencia y comportamientos asociados 
en Lima Metropolitana y Callao. Lima: Ministerio de Salud y Universidad Nacional Federico Villarreal.

Ansión, J., Lazarte, A., Matos, S., Rodríguez, J. y Vega-Centeno, P. (1998). Educación: la mejor herencia. Decisiones educativas y expectativas de los padres de familia. Una aproximación empírica. Lima: Pontificia Universidad Católica del Perú.

APRODEH-CEDAL (1996). Los derechos económicos, sociales y culturales en el Perú. Lima: autor.

Asociación Peruana de Estudios e Investigación para la Paz (1993). Violencia en la región andina: caso Perú. Lima: autor.

Banaji, M. R. y Prentice, D. (1994). The self in social contexts. Annual Review of Psychology, 45, 297-332.

Basadre, J. (2000). Historia de la República del Perú. Lima: Universidad Ricardo Palma.

Boris, H. N. (1993). Envy. Northvale, N J: Aronson.

Bullock, A. (1991). Hitler and Stalin. Parallel lives. Londres: Harper Collins.

Buntinx, G. (1998). ¿Por qué no vivo en el Perú? (Una generación después). Notas al sesgo de una encuesta. Márgenes. Encuentro y Debate, 11, 135-145.

Buss, D. M. (2000). The evolution of happiness. American Psychologist, $55,15-23$.

Callirgos, J. C. (1993). El racismo. La cuestión del otro (y de uno). Lima: DESCO.

Callirgos, J. C. (1997). El (poco discreto) encanto de la burguesía. Distancias sociales y discursos legitimadores en el Perú de hoy. Lima: Instituto de Defensa Legal.

Carranza, V. (2000). Globalización y crisis social en el Perú. Lima: Instituto Francés de Estudios Andinos y Universidad Ricardo Palma.

Castilla del Pino, C. (Comp.) (1994). La envidia. Madrid: Alianza Universidad.

Ciabattari, J. (1989). Will the 90's be the age of envy? Psychology Today, 47-50. 
Delacampagne, Ch. (1983). Racismo y occidente. Barcelona: Argos. Eguren, F., Cano, J. y del Aguila, A. (1997). Evaluación social del desarrollo humano en el Perú. Lima: Acción Ciudadana.

Elias, N. (1998). La civilización de los padres y otros ensayos. Barcelona: Norma.

Flores Galindo, A. (1988). Tiempo de plagas. Lima: El Caballo Rojo. Flores Galindo, A. (1997). Independencia y clases medias. En A. Flores Galindo (Ed.), Obras completas (vol. 5) (327-343). Lima: Sur. Foster, G. M. (1972). The anatomy of envy: a study in symbolic behavior. Current Anthropology, 13, 165-202.

Furnham, A., Borovoy, A. y Henley, S. (1986). Type A behaviour pattern, the recall of positive personality information and self-evaluations. British Joumal of Medical Psychology, 59, 365-374.

Girard, R. (1995). Shakespeare. Los fuegos de la envidia. Barcelona: Anagrama.

González Prada, M. (1976). Páginas libres y horas de lucha. Miranda: BibliotecaAyacucho.

Hansberg, O. (1996). La diversidad de las emociones. México DF: Fondo de Cultura Económica.

Harré, R. (1982). El ser social. Una teoría para la psicología social. Madrid: Alianza Universidad.

Henley, S. y Furnham, A. (1989). The type A behaviour pattern and self-evaluation. British Journal of Medical Psychology. 62, 5159.

Horrocks, J. E. (1984). Psicología de la adolescencia. México, DF: Trillas.

Izard, C. E. (1991). The psychology of human emotions. Nueva York: Plenum Press.

Klein, M. (1976). Envy and gratitude. Londres: Hogarth.

Klemperer, V. (1991). LTI. Notizbuch eines Philologen. Leipzig: Reclam-Verlag.

Kruse, A. y Schmitt, E. (2000). Wir haben uns als Deutsche gefühlt. Lebensrückblick und Lebenssituation jüdischer Emigranten und Lagerhäftlinge. Darmstadt: Steinkopff Verlag. 
Situaciones cotidianas anómalas y afectos negativos en el Perú

León, R. (1998a). El país de los extraños. Una encuesta sobre actitudes raciales en universitarios de Lima Metropolitana. Lima: Universidad Ricardo Palma.

León, R. (1998b). Situaciones de comparación social y afectos negativos en universitarios: un reporte de investigación. Persona, 1 , 163-177.

León, R., Delhonte, P., del Castillo, B. y León, M. (1998). La hipocresía en el Perú: una exploración. Más Luz. Revista Iberoamericana de Psicología y Pedagogía, 4, 77-304.

León, R. y Gómez Sánchez L., R. (1989). Resentimiento en adolescentes escolares de condición socio-económica alta y baja. $R e$ vista de Psicología, 6, 35-49.

León, R. y Martell, E. (1994). Valoración de conductas verbales y no verbales como expresión de envidia. Revista de Psicología, 12, 191-219.

León, R. y Moscoso, S. (1991). Percepción de la envidia en el Perú en un grupo de estudiantes universitarios de Lima Metropolitana. Revista de Neuro-psiquiatría, 54, 9-8.

León, R., Reyes, G. y Vela, O. (2000). Racismo, aristocracia y pseudomodernidad. Actitudes raciales en Lima y Trujillo. Trujillo: Universidad Ricardo Palma.

León, R. y Romero, C. (1990). Estudios acerca del resentimiento. Lima: Concytec.

León, R. y Tan, J.J. (1998). Humillados y ofendidos: un estudio acerca del desprecio y la discriminación en el Perú. Revista de Psicología, $16,45-81$.

Lersch, Ph. (1968). La estructura de la personalidad. Barcelona: Scientia.

López, S. (1997). Ciudadanos reales e imaginarios. Concepciones, desarrollo y mapas de ciudadanía en el Perú. Lima: Instituto de Diálogo y Propuesta.

Lora, C. (1994). Cultura y vida cotidiana. Páginas, 130, 36-41.

Lora, C. (2000). ¿Indiferencia o conciencia de las dificultades y de las frustraciones? Ideele, 125, 35. 
Manrique, N. (1996). Racismo y violencia política en el Perú. Pretextos, $8,89-105$.

Manrique, N. (1999). La piel y la pluma. Escritos sobre literatura, etnicidad y racismo.Lima: Sur.

Margalit, A. (1997). La sociedad decente. Barcelona: Paidós.

Matos Mar, J. (1987). Desborde popular y crisis del estado. El nuevo rostro del Perú en la década de 1980. Lima: Instituto de Estudios Peruanos.

Milobenski, E. (1964). Der Neid in der griechischen Philosophie. Wiesbaden: Harrassowitz.

Milla Batres, C. (Ed.) (1990). En qué momento se jodió el Perú. Lima: Milla Batres.

Nevo, O., Nevo, B. y Derech-Zehavi, A. (1994). The tendency to gossip as a psychological disposition. En R. F. Goodman y A. Ben-Ze'ev (Eds.), Good gossip (pp. 180-192). Lawrence: University Press of Kansas.

Nietzsche, F. (1983). La genealogía de la moral. Madrid: Alianza Editorial.

Nugent, J. G. (1992). El laberinto de la choledad. Lima: Fundación Friedrich Ebert.

Panfichi, A. y Portocarrero, F. (Eds) (1995). Mundos interiores. Lima, 1850-1950. Lima: Universidad del Pacífico.

Pásara, L. y Parodi, J. (Eds) (1988). Democracia, sociedad y gobierno en el Perú. Lima: Centro de Estudios de Democracia y Sociedad.

Pedraglio, S. (2001). Los monederos falsos. Quehacer, 132, 56-61.

Plutchik, R. (1997). Las emociones. México, DF: Diana.

Portocarrero, G. (1990). Violencia estructural en el Perú: sociología. Lima: Asociación Peruana de Estudios e Investigación para la Paz.

Portocarrero, G. (1993). Racismo y mestizaje. Lima: Sur.

Portocarrero, G. (Ed) (1998). Las clases medias: entre la pretensión y la incertidumbre. Lima: Oxfam-Sur.

Romero, C. (2001). Viviendo con el enemigo. La confianza en los otros en el Perú. Páginas, 168, 57-66 
Situaciones cotidianas anómalas y afectos negativos en el Perú

Romero, C. y Sulmont, D. (2000). El estudio de los valores en el Perú. Debates en Sociología, 25-26, 245-281.

Rovaletti, M. L. (2000). Identidad y esquizofrenia. Los personajes en busca de un autor. Analogía Filosófica. Revista de Filosofía, 14 (2), 211-223.

Russell, B. (1978). La conquista de la felicidad. Madrid: Espasa Calpe.

Salovey, P. (Ed.) (1991). The psychology of jealousy and envy. Londres: The Guilford Press.

Santos Anaya, M. (1999). ¿Sabes con quién estás hablando? Un ensayo sobre la dinámica individuo-persona en la sociedad peruana. Lima: Instituto de Defensa Legal.

Scheler, M. (1944). El resentimiento en la moral. Buenos Aires: Espasa Calpe.

Spaks, P. M. (1985). Gossip. Nueva York: Knopf.

Stein, S. y Monge, C. (1988). La crisis del estado patrimonial en el Perú. Lima-Miami: Instituto de Estudios Peruanos y Universidad de Miami.

Tello, M. P. (1989). Sobre el volcán. Diálogo frente a la subversión. Lima: Centro de Estudios Latinoamericanos.

Tomei, P. (1995). Envidia en las organizaciones. Minimice sus repercusiones aprendiendo a manejarla. México, DF: McGraw Hill.

Twanama, M. (1992). Cholear en Lima. Márgenes, 5 (9), 206-240.

Vargas Durand, L. (1995). Martín Adán. Lima: Brasa.

Vargas Llosa, M. (1993). El pez en el agua. Memorias. Barcelona:

Seix Barral.

Vinaccia, S., Tobón, S., Sandín, B. y Martínez Sánchez, F. (2001).

Estrés psicosocial y úlcera péptica duodenal: una perspectiva bio-psico-social. Revista Latinoamericana de Psicología, 33, 117-130.

Walcot, P. (1978). Envy and the Greeks. A study of human behavior. Warminster: Aris \& Phillips.

Westacott, E. (sin fecha). La confianza interpersonal en el Perú. Lima: ESAN. 
Ramón León

Youngers, C. (1999-2000). Para entender la política de los Estados Unidos hacia el Perú. Ideele, 124, 106-109. 


\section{Anexo}

\section{Instrumentos}

1. La Lista de Evaluación de Frecuencia de Afectos y Conductas Sociales estuvo conformada por ocho afectos y conductas negativos, y dos positivos. Los negativos fueron: (a) sentir envidia; (b) experimentar resentimiento; (c) agredir verbalmente a otro (o ganas de hacerlo); (d) agredir físicamente a otro (o ganas de hacerlo); (e) mostrarse indiferente; (f) ser despreciativo; (g) tener prejuicios raciales; y, (h) hablar mal de los demás. Los positivos: (a) confiar en los demás; y, (b) ser solidario.

Los afectos y conductas negativos suelen ser considerados como frecuentes en el Perú. La envidia ha sido anotada por Basadre (2000) y estudiada en recientes trabajos (León y Martell, 1994; León y Moscoso, 1991); el resentimiento ha sido reconocido como una realidad de gran intensidad y estudiada asimismo en varios trabajos (León y Gómez Sánchez, 1989; León y Romero, 1990); las agresiones verbales y físicas se pueden verificar en los anales judiciales y en la violencia cotidiana en el país; la indiferencia y el desprecio han sido observados y descritos (Nugent, 1992; León y Tan, 1998), así como los prejuicios raciales (Callirgos, 1993; León, 1998a; León et al., 2000; Portocarrero, 1993). La maledicencia, anotada por observadores de la realidad peruana (Vargas Durand, 1995; Vargas Llosa, 1993), apenas ha sido estudiada.

La Lista de Evaluación de Frecuencia de Afectos y Conductas Sociales explora la frecuencia con la que las personas experimentan (y creen que los demás experimentan) determinadas emociones referidas a su vida de relación interpersonal, tales como la envidia, el desprecio o la solidaridad. 
Esta exploración es, por supuesto, sumamente problemática. En primer lugar porque las personas no suelen ser objetivas en la valoración de su propia vida afectiva, como tampoco lo son cuando interpretan la de los demás. Debe agregarse a esto que la Lista incluye ocho afectos negativos (por ejemplo, la envidia y la maledicencia) que difícilmente suelen ser admitidos como presentes en uno mismo (Nevo, Nevo y Derech-Zehavi, 1994; Spaks, 1985). Esto último debe ser tomado en consideración al momento de analizar los resultados.

La lista solicita que se valore la frecuencia (en seis posibilidades: 0 , nunca; 1 , casi nunca; 2 , pocas veces; 3 , con regular frecuencia; 4 , con mucha frecuencia; y, 5, siempre) de diez afectos y conductas sociales en él mismo (en ti), en su entorno inmediato (en tu barrio) y en la sociedad peruana.

El área en ti es un reactivo de autorreporte. Es en ella en la que puede presumirse la mayor presencia de numerosos sesgos que afectan a la autopercepción. Como se sabe, algunas personas tienden a valorarse de modo negativo en unos casos (Henley y Furnham, 1989), pero en otros, como parece el caso de los afectos sociales negativos, adaptan sus autovaloraciones a las expectativas sociales (Nevo et al. 1994).

El área en tu barrio, como ya se dijo, solicita que la persona evalúe la frecuencia de los diez afectos y conductas en su entorno social inmediato, así como en el área en la sociedad peruana, se le pide lo mismo con referencia a la sociedad peruana.

El barrio es el contexto físico y social más inmediato de las personas; aquel con el cual tiene mayor familiaridad y con el que puede haber desarrollado inclusive intensos vínculos afectivos. En ese contexto se encuentran los vecinos, amigos, conocidos, compañeros de escuela y de universidad, y hasta de trabajo. Se trata, por tanto, del escenario de frecuentes interacciones sociales. 
En lo referido a la sociedad peruana, se trata más bien de la percepción que los sujetos tienen de la realidad social del Perú.

Los puntajes de estas dos áreas conjugan estereotipos, creencias, suposiciones y experiencias personales. Obviamente, no se puede precisar la magnitud de la influencia de cada uno de ellos: los puntajes son indicadores gruesos de la percepción que las personas tienen de la vida de relación social en el Perú. El Cuadro 1 presenta las correlaciones ítem-total y el alfa de Crombach de cada una de las áreas.

\section{Cuadro 1}

Correlación ítem-total y alfa de Crombach de los afectos y conductas en las áreas $t i$ mismo, barrio y sociedad peruana

\begin{tabular}{|lccc|}
\hline \multicolumn{1}{|c}{ Situación } & Ti mismo & Barrio & Sociedad peruana \\
\hline Envidia & .45 & .62 & .73 \\
Resentimiento & .44 & .62 & .65 \\
Agresión Verbal & .46 & .62 & .72 \\
Agresión Física & .38 & .61 & .73 \\
Indiferencia & .40 & .44 & .62 \\
Desprecio & .50 & .65 & .75 \\
Prejuicios sociales & .46 & .56 & .76 \\
Hablar mal de los demás & .46 & .60 & .70 \\
Confianza en los demás & .01 & .16 & .20 \\
Solidaridad &. .05 & .02 & -.13 \\
\hline \multicolumn{1}{|c}{ alfa } & .68 & .81 & .86 \\
\hline
\end{tabular}

Los ocho afectos y conductas negativos tuvieron adecuadas correlaciones item-total, a diferencia de confiar en los demás y ser solidario, que arrojaron correlaciones item-total bajas. Por su parte, los alfa de las tres áreas fueron elevados. En una nueva correlación itemtotal de sólo los afectos y conductas negativos, se obtuvieron correlaciones más elevadas; lo mismo sucedió con los alfa. Se evaluó asimismo las correlaciones item-total de cada uno de los afectos y conductas de la Lista (Cuadro 2). 


\section{Cuadro 2}

Correlaciones item-total y alfa de Crombach de los afectos y conductas, según el ámbito evaluado: ti mismo, barrio, sociedad peruana

\begin{tabular}{|c|c|c|c|c|}
\hline \multirow[t]{2}{*}{ Situación } & \multicolumn{3}{|c|}{ Área } & \multirow[t]{2}{*}{ alfa } \\
\hline & En ti & Barrio & Sociedad peruana & \\
\hline Envidia & .38 & .55 & .50 & .66 \\
\hline Resentimiento & .28 & .53 & .42 & .59 \\
\hline Agresión Verbal & .39 & .52 & .50 & .65 \\
\hline Agresión Física & .27 & .55 & .43 & .60 \\
\hline Indiferencia & .43 & .59 & .49 & .69 \\
\hline Desprecio & .38 & .65 & .52 & .68 \\
\hline Prejuicios sociales & .36 & .63 & .48 & .66 \\
\hline Hablar mal de los demás & .34 & .65 & .61 & .70 \\
\hline Confianza en los demás & .23 & .53 & .33 & .54 \\
\hline Solidaridad & .35 & .54 & .42 & .62 \\
\hline
\end{tabular}

Todas las correlaciones estuvieron por encima de .30 , con la excepción de sentir resentimiento, agresión física y confiar en otros en el área en ti. Los alfa fueron aceptables, si se tiene en cuenta que se trata de listas de sólo tres ítems.

2. La Lista de Situaciones Anómalas estaba compuesta por diez ítems: (a) pagar una coima; (b) ser discriminado por mi raza; (c) ser víctima de un robo; (d) ser objeto de chismes; (e) ser insultado; (f) sentir que se me desprecia; ( $g$ ) no se reconocen mis méritos; (h) ser víctima de un engaño; (i) ser tratado con indiferencia; y, (j) ser agredido físicamente.

Las situaciones cotidianas tienen, como su nombre lo da a entender, una ocurrencia diaria y «consisten en la experimentación objetiva de eventos en la vida cotidiana que pueden ser positivos o negativos, o tener algo de negativos y positivos al mismo tiempo, de acuerdo a la percepción que haga el sujeto de ellos» (Vinaccia, Tobón, Sandín y Martínez, 2001; p. 119). Por situación anómala entendemos un contexto social en el cual una o más personas sienten que 
su integridad física, imagen social, y sus derechos y su persona han sido ignorados, violados o están a punto de serlo.

La lista explora la ocurrencia de diez situaciones cotidianas anómalas a través de dos posibilidades (si-no), y la posibilidad de que causen cuatro afectos: resentimiento, temor, cólera y vergüenza. En la evaluación de cada uno de los afectos se solicitó a los sujetos que escogieran una alternativa entre 0 (nada) y 5 (mucho).

Las diez situaciones que forman la lista suelen ser consideradas frecuentes en el Perú. Sin embargo, para la gran mayoría de ellas no existen estadísticas ni estudios específicos. Algunas pueden ser valoradas de modo objetivo, como pagar una coima o ser víctima de un robo. Otras, sin embargo, son eminentemente subjetivas: ser objeto de chismes, ser tratado con indiferencia o sentir que alguien me desprecia.

El Cuadro 3 presenta las correlaciones item-total para las diez situaciones en cada uno de los cuatro afectos. Los resultados son significativos.

\section{Cuadro 3}

Correlaciones ítem-total y alfa de Crombach de cada uno de los cuatro afectos

\begin{tabular}{|lcccc|}
\hline \multicolumn{1}{|c}{ Situación } & Resentimiento & Temor & Cólera & Vergüenza \\
\hline 1. Pagar una coima & .34 & .35 & .39 & .19 \\
2. Ser discriminado & .56 & .49 & .52 & .51 \\
3. Ser víctima de robo & .39 & .33 & .40 & .51 \\
4. Ser objeto de chismes & .64 & .62 & .51 & .58 \\
5. Ser insultado & .65 & .64 & .57 & .56 \\
6. Ser despreciado & .62 & .63 & .53 & .62 \\
7. No se reconocen mis méritos & .57 & .61 & .54 & .49 \\
8. Ser víctima de un engaño & .55 & .63 & .58 & .52 \\
9. Ser tratado con indiferencia & .64 & .68 & .59 & .61 \\
10. Ser agredido físicamente & .57 & .46 & .51 & .60 \\
\hline \multicolumn{1}{|c}{ alfa } & .85 & .84 & .83 & .82 \\
\hline
\end{tabular}


Asimismo, se calcularon las correlaciones ítem total para los cuatro afectos en cada una de las situaciones (Cuadro 4). Todas las correlaciones fueron elevadas, así como lo son también los alfa.

\section{Cuadro 4}

Correlaciones ítem-total y alfa de Crombach de los afectos en las situaciones de la lista de situaciones anómalas

\begin{tabular}{|lccccc|}
\hline \multicolumn{1}{|c}{ Situación } & \multicolumn{3}{c}{ Afecto } & \multicolumn{2}{c|}{ alfa } \\
& Resentimiento & Temor & Cólera & Vergüenza \\
\hline 1. Pagar una coima & .45 & .51 & .48 & .48 & .71 \\
2. Ser discriminado & .56 & .40 & .52 & .42 & .69 \\
3. Ser víctima de robo & .40 & .36 & .47 & .13 & .54 \\
4. Ser objeto de chismes & .50 & .45 & .44 & .46 & .68 \\
5. Ser insultado & .52 & .34 & .44 & .40 & .65 \\
6. Ser despreciado & .62 & .47 & .53 & .47 & .74 \\
7. No se reconocen mis méritos & .52 & .37 & .48 & .30 & .65 \\
8. Ser víctima de un engaño & .45 & .35 & .45 & .28 & .60 \\
9. Ser tratado con indiferencia & .55 & .43 & .54 & .46 & .71 \\
10. Ser agredido físicamente & .62 & .47 & .54 & .35 & .71 \\
\hline
\end{tabular}

En resumen, los resultados presentados en las tablas previas permiten afirmar que las propiedades psicométricas de ambas listas son adecuadas para el desarrollo del trabajo de investigación aquí reportado.

3. Lista de sentimientos que contribuyen al subdesarrollo del Perú (que incluía el resentimiento, la desconfianza entre los peruanos, la envidia, el racismo y la falta de solidaridad).

4. Lista de causas del resentimiento y de la envidia en el Perú (con cinco posibilidades: diferencias económicas entre los peruanos; falta de oportunidades para los peruanos; los peruanos somos así; mucho racismo y discriminación entre los peruanos; y mucho desprecio entre los peruanos). 
Situaciones cotidianas anómalas y afectos negativos en el Perú

El siguiente Cuadro muestra las correlaciones item-total y los alfa correspondientes obtenidos a través de la lista de causas de resentimiento y envidia.

\section{Cuadro 5}

Correlación ítem-total y alfa de Crombach de la lista de causas de resentimiento y envidia

\begin{tabular}{|lcc|}
\hline \multicolumn{1}{|c|}{ Causas } & Resentimiento & Envidia \\
\hline Diferencias económicas entre los peruanos & .41 & .41 \\
Falta de oportunidades para los peruanos & .38 & .46 \\
Los peruanos somos así & .24 & .30 \\
Racismo y discriminación entre los peruanos & .48 & .54 \\
Desprecio entre los peruanos & .49 & .54 \\
\hline \multicolumn{1}{|c|}{ alfa } & .63 & .69 \\
\hline
\end{tabular}

